\title{
BMJ Open Effects of local handwashing agents on microbial contamination of hands in the rural settings of northwest Ethiopia: protocol for a two-arm, clustered- randomised controlled trial
}

\author{
Zemichael Gizaw (D) , ${ }^{1,2,3}$ Alemayehu Worku Yalew, ${ }^{4}$ Bikes Destaw Bitew, ${ }^{1}$ \\ Jiyoung Lee, ${ }^{5,6}$ Michael Bisesi ${ }^{5}$
}

To cite: Gizaw Z, Yalew AW, Bitew BD, et al. Effects of local handwashing agents on microbial contamination of hands in the rural settings of northwest Ethiopia: protocol for a two-arm, clustered-randomised controlled trial. BMJ Open 2021;11:e046828. doi:10.1136/ bmjopen-2020-046828

- Prepublication history for this paper is available online To view these files, please visit the journal online (http://dx.doi org/10.1136/bmjopen-2020046828).

Received 12 November 2020 Accepted 19 July 2021

Check for updates

(c) Author(s) (or their employer(s)) 2021. Re-use permitted under CC BY-NC. No commercial re-use. See rights and permissions. Published by BMJ.

For numbered affiliations see end of article.

Correspondence to

Zemichael Gizaw;

zemichael12@gmail.com

\section{ABSTRACT}

Introduction Hands play a part in the transmission of infections. Handwashing with soap sufficiently reduces the level of hand contamination and the spread of infections. As soap is not usually available due to cost, ash is often used as a zero-cost alternative to soap in the rural settings of developing countries. However, there is limited evidence on the effectiveness of ash to reduce microbial contamination of hands. This study is, therefore, designed to assess the effect of ash on microbial contamination of hands in the rural settings of northwest Ethiopia.

Methods and analysis A two-arm clustered-randomised controlled trial will be employed. A total of 11 clusters per arm will be selected using simple random sampling technique. A total of 220 mothers or caregivers of under-5 children will be included in each arm. After providing health education on effective handwashing process, we will ask study subjects to do the usual activities. We will then take swab samples from the dominant hand before washing. After swabbing, participants will be asked to wash their hands with water only and with ash by following effective handwashing procedures. We will again take swab samples from the dominant hand after washing and drying. Finally, we will compare each intervention arm against the control. A generalised estimating equation (GEE) with robust SE estimation will be used to account the cluster nature of data.

Ethics and dissemination Results will be published in peer-reviewed journal and presented at international conferences. The protocol is approved by the Institutional Review Board of the University of Gondar, Ethiopia. Trial registration number Pan African Clinical Trial Registry; PACTR202011855730652.

\section{INTRODUCTION}

It is universal that the practice of good hand hygiene is one of the easiest and most important things that humans can do for themselves in their daily lives to reduce the risks of infection. Hands are especially important given that they are the last line of defence within the chain of transmission
Strengths and limitations of this study

- This study will assess the effect of ash on microbial contamination of hands in the rural settings of northwest Ethiopia as there is limited evidence on the effect of ash on microbial contamination of hands in Ethiopian context.

- To control bias, clusters will be randomly allocated to the control and intervention arms; a buffer zone of a minimum of 15 min walking distance will be used before enrolling the next cluster; and principal investigators will be remaining blinded to the randomised group assignments until the primary analysis is completed.

- As a limitation, in this study, we will not evaluate the $\mathrm{pH}$ and microbial quality of ashes, we will use for the trial; however, we will use freshly produced ash since freshly produced ash through the burning of wood is sterile to minimise cross-contamination of hands from the ash.

of gastrointestinal pathogens, either directly from hand to mouth or indirectly via the handling of food or water. Hands can also play a role in the transmission of respiratory tract infections where fingers turn out to be infected with breathing viruses, contamination can be transferred by way of rubbing the conjunctiva of the eyes or the nasal mucosa. The hands also can play a part in the transmission of skin, wound, eye and other infections. ${ }^{12}$

There is a clear causal link between hand hygiene and infection transmission. Hands are one of the most important mechanisms to transmit infection. ${ }^{3}$ Evidence indicates that hands together with food contact or other environmental surfaces cause $60 \%$ of the spread of gastrointestinal infection. Contaminated hands could also cause up to $50 \%$ of 
respiratory tract infections. ${ }^{4}$ Promotion of improved hand hygiene has been recognised as an important public health measure. Results from intervention studies show that handwashing reduces gastrointestinal and respiratory tract infections up to $50 \%-60 \%{ }^{4}$ Handwashing education in the community reduces the number of people who get sick with diarrhoea by $23 \%-40 \%^{5-7}$; reduces diarrheal illness in people with weakened immune systems by $58 \%^{8}$; reduces respiratory illnesses, like colds, in the general population by $16 \%-21 \%^{69}$ and reduces absenteeism due to gastrointestinal illness in schoolchildren by $29 \%-57 \% .^{10}$

Microorganisms are removed from the hands by the mechanical action of rubbing and rinsing rather than killed by a special handwashing preparation. ${ }^{11-13}$ The effectiveness of rubbing in the removal of microbes is determined by the degree to which microbes are bound to the surface of the skin. The use of soap greatly improves the elimination of bacteria on the hands compared with that accomplished by washing with water alone. In lowincome societies in developing countries, soil or mud and ash are sometimes used as a zero-cost alternative to handwashing soap. ${ }^{4}$ For instance, studies conducted in Bangladesh and India found that soil, ash and soap were all effective in removing faecal coliforms from hands. ${ }^{14} 15$ However, there is limited evidence on the effect of ash on microbial contamination of hands in Ethiopian context. This community-based clustered-randomised controlled trial is, therefore, designed to assess the effect of ash on microbial contamination of hands among mothers or caregivers of under- 5 children in the rural settings of northwest Ethiopia.

\section{Research question and objectives}

Is handwashing with ash effective to reduce the burden of microorganisms on hands in northwest Ethiopia?

Use of soap and other alcohol-based agents significantly increases the reduction of microbes on hands relative to that achieved by washing with water alone. As soap-based or alcohol-based agents are not usually available due to cost, ash is often used as a zero-cost alternative to soap for handwashing in the rural settings of developing countries. ${ }^{4}$ However, there is limited evidence on the effect of ash on microbial contamination of hands in Ethiopian context. This study is, therefore, designed to assess the effect of local handwashing agents, mainly ash on microbial contamination of hands among mothers or caregivers of under- 5 children in the rural settings of northwest Ethiopia.

\section{METHODS}

\section{Study area and settings}

This study was carried out in the rural settings of east Dembiya district. East Dembiya is one of the 13 woredas in central Gondar zone, the Amhara national regional state, Ethiopia. East Dembiya is bordered on the south by Lake Tana, on the west by west Dembiya, on the north by Lay Armachiho and on the east by Gondar Zuria and Gondar town. The district is subdivided into 28 rural and 4 urban kebeles (the lowest administrative unit). ${ }^{16}$ The district health department report in July 2020 showed that the east Dembiya district had a total population of 210761 , of whom 192020 (91\%) and 18741 (9\%) were rural and urban residents, respectively. Of a total population in the district, 104327 were men and 106434 were women with 1:1 sex ratio. ${ }^{16}$ Hygiene and sanitationrelated communicable diseases were highly prevalent in the area. During June 2017, intestinal parasitic infections and diarrheal disease were the top four and five prevalent diseases, which accounted $5161(9.97 \%)$ and 4981 $(9.62 \%)$, respectively. ${ }^{17}$ The population in the area had poor access to sanitation. During June 2017, clean water and latrine coverage in the district was $26.60 \%$ and $55 \%$, respectively. ${ }^{17}$

\section{Study design}

A two-arm clustered-randomised non-crossover trial will be employed among mothers or caregivers of under-5 children in the rural settings of northwest Ethiopia to see the effect of local handwashing agents on bacterial contamination of hands.

\section{Participant eligibility and enrolment}

This trial will be implemented among mothers of under-5 children in east Dembiya district, northwest Ethiopia, where no similar interventions are not operating or planned in the intervention period. Participants will be recruited based on the following inclusion criteria: (a) presence of under-5 children in the household because we will link the results of this trial as a factor for another study titled exposure to enteric infection and environmental enteric dysfunction in under-5 children in northwest Ethiopia, (b) mothers or caregivers who are volunteer to be part of the experiment, (c) mothers or caregivers who are not using soap to wash hands and (d) geographical accessibility. Mothers or caregivers having acute or chronic nail or skin disorders/lesions, including dermal abrasion, trauma and infection, will be excluded.

\section{Description of interventions}

In this study, we will provide health education to both arms on effective handwashing procedures and local rubbing agents like ash to the intervention groups. The control arm will not use ash. Controls will be those mothers or caregivers who continue with their usual practice.

\section{Sample size determination}

Stata V.14 is used to calculate the number of clusters with the following assumptions: $\log _{10}$ mean bacterial count before handwashing (right hand) with $\mathrm{SD}=3.17 \pm 0.71$; $\log _{10}$ mean bacterial count after handwashing (right hand) with $\mathrm{SD}=3.41 \pm 0.61^{18}$; equal cluster size with average size $(\mathrm{m})=20$; intracluster correlation coefficient $\left(\right.$ ICC) $=0.02$ as recommended by Hayes and Bennett ${ }^{19}$; $80 \%$ power; $95 \%$ CI with a $5 \%$ alpha level. With these assumptions, the number of clusters per arm becomes 
10 and design effect $(1+(m-1)$ ICC) of 1.38. Assuming one extra cluster for loss of follow-up, the total cluster becomes 11 per arm. Therefore, a total of 220 mothers or caregivers will receive the intervention and 220 mothers or caregivers will be taken as control groups.

\section{Strategies for achieving adequate participant enrolment}

Participants will be informed about the purpose and procedures of the study and will be recruited after we received informed consent. We will not enforce participants to be part of this study. However, we will use different mechanisms (for instance, providing soap for handwashing after the trial is completed) to increase participation.

\section{Sampling procedures}

The number of clusters will be selected based on simple random sampling method. Clusters will be defined by the presence of adequate households with under-5 children eligible for enrolment and geographical accessibility. We will conduct a pilot survey to determine the size of the clusters. As indicated in the sample size calculation section, the geographical area where we will get households with 20 under-5 children will be taken as a cluster. A buffer zone of a minimum of $15 \mathrm{~min}$ walking distance will be used before enrolling the next cluster, to minimise information contamination between enrolled clusters. Adjacent clusters will be defined to form a block and clusters will be randomly assigned to the study arms within the block. All households with under-5 children in the selected clusters and who fulfilled the inclusion criteria will enter into clustered-randomised controlled trial schemes.

\section{Randomisation and blinding}

Clusters will be randomly allocated in each arm. The random sequence generation and allocation will be conducted by the coordinating team with a reproducible seed. Due to the nature of the interventions and the study purpose, participants will not be blinded to their intervention assignment. Principal investigators will be remaining blinded to the randomised group assignments until the primary analysis is completed.

\section{Outcome measures}

The primary outcome of this study is microbial contamination of hands, measured by mean concentration of indicator organism for faecal contamination, that is, Escherichia coli before and after washing hands with water alone and with ash. We adopted a procedure to recover E. coli from the standard methods described in United Nations Environment Programme (UNEP) and WHO guidelines. ${ }^{2021}$

\section{Data collection tools and procedures}

After providing health education on effective handwashing process, we will ask study subjects to do the usual activities. We will then take swab samples from the dominant hand before washing. After swabbing, participants will be then asked to wash their hands with water only and with ash by following effective handwashing procedures with instructions on length of time or thoroughness and will be informed to dry their hands in the air. We will again take swab samples from the dominant hand after washing and drying. In total, 880 swab samples will be collected and analysed for bacteria. Observational checklists will be used to observe sanitation condition of the living environment. The presence and type of domestic animals and their faeces will be also recorded. We will also check the microbial quality of the ash to check whether ash itself causes microbial contamination or not.

\section{Schedule of enrolment, interventions, assessments and visits for participants}

Table 1 shows detail of schedule of enrolment, interventions, assessments and visits for participants.

\section{Differential attrition (loss to follow-up)}

We will be closely tracking participants to minimise attrition. To assess if attrition is random, we will compare attrition rates across randomised arms and also the characteristics of those lost to follow-up versus those remain. We will do sensitivity analysis using worst-case imputation bounds for our effect estimates ${ }^{22}$ and we will also calculate bounds if we find a systematic attrition that is not

Table 1 Schedule of enrolment, intervention and assessment 
balanced across arms. We will also use semiparametric weighting using baseline characteristics if attrition is high.

\section{Differential missing outcome measurements}

We will carefully track enrolled participants and the completeness of samples per participant. We will compare rates of missing samples across randomised arms and also the characteristics of those with missing versus those with a full set of samples to determine whether missing is random or not. If we find that missing outcomes are imbalanced across arms, we will impute using appropriate method for imputation that is based on drawing from the observed distribution.

\section{Data management and statistical analysis}

Data will be checked for completeness during the time of collection. Data will be entered and coded in Epi-info V.7. To improve quality, double data entry will be considered. Data will be analysed using Stata V.14. Descriptive statistics of variables will be reported as proportion or mean and SD. In our analysis, we will compare intervention arm against the control arm in an intention to treat analysis. We will have two comparisons: handwashing with water only versus bacterial contamination of hands and handwashing with ash versus bacterial contamination of hands.

We will estimate adjusted parameters by including variables that are associated with the outcome variable to improve the precision of our estimates (decrease the SEs). A GEE with robust SE estimation will be used for the analysis to account the cluster nature of data. The link function will be determined based on the type and distribution of data. The working correlation structure will be determined based on the results of Quasi-likelihood information criterion (QIC) analysis. Model with the smallest QIC will be chosen.

\section{Interim analysis}

We will not conduct interim outcome analysis that includes information about randomised assignments until all of the data from all the study subjects are collected.

\section{Data quality management}

This study will be employed after the soundness of the methodology is approved by senior researchers and experts in the area. The data collection tool will be tested for validity and reliability to gather valid and reliable information. The data collection will be held by experienced and trained field data collectors. Trained laboratory technologists will be participated to collect swab samples. All aseptic measures will be considered during sample collection, sample transportation and analysis as stated in the WHO (2011) Laboratory Quality Management System Handbook. ${ }^{23}$ The shelf-life of laboratory reagents and kits will be checked before use. Field supervisors will closely supervise the data collection process and will check the completeness of the filled questionnaire. The principal investigator will closely supervise the laboratory procedures to maximise the quality of the analysis. Moreover, field supervisors will audit the implementation and assessment of interventions. The principal investigator and field supervisors will have a short meeting daily to discuss on problems or challenges data collectors will face in each day of data collection. Inconsistencies and other problems in the implementation and assessment of interventions will be solved.

\section{Roles and responsibilities of sponsor organisations}

The sponsor organisations will have no roles in study design; collection, management, analysis and interpretation of data; writing of the report and the decision to submit the report for publication.

\section{Plans for communicating important protocol modifications}

Important changes to the protocol will be communicated to the funders and have to be agreed on and will be communicated with the institutional review board. Modifications of the intervention are expected to be part of the implementation process and will be consented and communicated during the kick-off-meetings. Finally, for any modification, the edited version will be submitted to Pan African Clinical Trial Registry.

\section{Patient and public involvement}

No patient was involved.

\section{ETHICAL CONSIDERATION}

\section{Authorisation}

Ethical clearance was obtained from the institutional review board of the University of Gondar and official letters will be submitted to the local district administrators and health departments.

\section{Protection of human subject}

The data collection tools will be proved not to affect the morale and personality of study subjects. The materials to be used only for this research purpose will not have any health hazards. There will be no risk in participating in this research project, and the collected data will be used only for this research purpose.

\section{Informed consent}

Informed consent will be obtained from household heads or mothers.

\section{Privacy and confidentiality}

We will assure privacy and confidentiality. No identifiers will be included in the data collection tools. All information collected from study subjects during the course of the research will be kept strictly confidential. Any information about study subject will not be linked with names and will be accessed only by the researchers.

\section{Benefit of the study}

Study subjects will not receive financial benefits for their participation. Data collectors will provide oral rehydration solutions for children who have diarrhoea at the 
time of data collection. We will also advise mothers to visit health facilities for further management.

\section{Incentives/payment for participating}

There is no incentive or payment to be gained by taking part in this project. However, participants may receive expenses for transport, meal and compensation for loss if they are referred to the district health centres for the research purpose. We cannot promise that the study will directly help study subjects, but the information we get from the study will help to know the effect of washing hands with ash on microbial contamination.

\section{Author affiliations}

${ }^{1}$ Department of Environmental and Occupational Health and Safety, Institute of Public Health, College of Medicine and Health Sciences, University of Gondar, Gondar, Ethiopia

${ }^{2}$ Global One Health Initiative(GOHi), The Ohio State University, Columbus, Ohio, USA

${ }^{3}$ Addis Continental Institute of Public Health, Addi Ababa, Ethiopia, Addis Ababa, Ethiopia

${ }^{4}$ School of Public Health, Addis Ababa University, Addis Ababa, Ethiopia

${ }^{5}$ Division of Environmental Health Sciences, College of Public Health, The Ohio State University, Columbus, Ohio, USA

${ }^{6}$ Department of Food Science and Technology, The Ohio State University, Columbus, Ohio, USA

Acknowledgements Authors acknowledge the University of Gondar, Addis Continental Institute of Public Health, One Health Eastern Africa Research Training (OHEART) programme at the Ohio State University, Global One Health Initiative(GOHi) and National Institutes of Health $(\mathrm{NIH})$ Fogarty International Centre for their support.

Contributors ZG prepared the protocol. AWY, BD, JL and MB reviewed the draft protocol. All the authors read and approved the final manuscript.

Funding The cost for laboratory reagents will be covered by One Health Eastern Africa Research Training (OHEART) programme at the Ohio State University, Global One Health Initiative (GOHi) through National Institutes of Health (NIH) Fogarty International Centre (grant number TW008650).

Competing interests None declared.

Patient and public involvement Patients and/or the public were involved in the design, or conduct, or reporting, or dissemination plans of this research. Refer to the Methods section for further details.

Patient consent for publication Not required.

Provenance and peer review Not commissioned; externally peer reviewed.

Open access This is an open access article distributed in accordance with the Creative Commons Attribution Non Commercial (CC BY-NC 4.0) license, which permits others to distribute, remix, adapt, build upon this work non-commercially, and license their derivative works on different terms, provided the original work is properly cited, appropriate credit is given, any changes made indicated, and the use is non-commercial. See: http://creativecommons.org/licenses/by-nc/4.0/.

ORCID iD

Zemichael Gizaw http://orcid.org/0000-0002-6713-1975

\section{REFERENCES}

1 Bloomfield SF, Aiello AE, Cookson B, et al. The effectiveness of hand hygiene procedures in reducing the risks of infections in home and community settings including handwashing and alcohol-based hand sanitizers. Am J Infect Control 2007;35:S27-64.

2 Fischler GE, Fuls JL, Dail EW, et al. Effect of hand wash agents on controlling the transmission of pathogenic bacteria from hands to food. J Food Prot 2007;70:2873-7.

3 Pittet D, Allegranzi B, Sax H, et al. Evidence-Based model for hand transmission during patient care and the role of improved practices. Lancet Infect Dis 2006;6:641-52.

4 Bloomfield SF, Nath KJ. Use of ash and mud for handwashing in low income communities. The International scientific forum on home hygiene (IFH), 2009. Available: http://www.ifh-homehygiene.org/ best-practice-review/use-ash-and-mud-handwashing-lowincomecommunities [Accessed 02 Jul 2021].

5 Ejemot-Nwadiaro RI, Ehiri JE, Meremikwu MM, et al. Hand washing for preventing diarrhoea. Cochrane database of systematic reviews 2008:1.

6 Aiello AE, Coulborn RM, Perez V, et al. Effect of hand hygiene on infectious disease risk in the community setting: a meta-analysis. $A m$ J Public Health 2008;98:1372-81.

7 Freeman MC, Stocks ME, Cumming O, et al. Hygiene and health: systematic review of handwashing practices worldwide and update of health effects. Trop Med Int Health 2014;19:906-16.

8 Huang DB, Zhou J. Effect of intensive handwashing in the prevention of diarrhoeal illness among patients with AIDS: a randomized controlled study. J Med Microbiol 2007;56:659-63.

9 Rabie T, Curtis V. Handwashing and risk of respiratory infections: a quantitative systematic review. Trop Med Int Health 2006;11:258-67.

10 Wang Z, Lapinski M, Quilliam E, et al. The effect of hand-hygiene interventions on infectious disease-associated absenteeism in elementary schools: a systematic literature review. Am J Infect Control 2017;45:682-9.

11 Sprunt K, Redman W, Leidy G. Antibacterial effectiveness of routine hand washing. Pediatrics 1973;52:264-71.

12 Lowbury EJ, Lilly HA, BULL JP. Disinfection of hands: removal of transient organisms. Br Med J 1964;2:230-3.

13 Paulson DS, Fendler EJ, Dolan MJ, et al. A close look at alcohol gel as an antimicrobial sanitizing agent. Am J Infect Control 1999;27:332-8.

14 Hoque BA, Mahalanabis D, Alam MJ, et al. Post-defecation handwashing in Bangladesh: practice and efficiency perspectives. Public Health 1995;109:15-24.

15 Anuradha P, Yasoda Devi P, Prakash MS, Devi PY. Effect of handwashing agents on bacterial contamination. Indian J Pediatr 1999;66:7-10.

16 Eshetie M. East Dembiya district health department plan and monitoring office annual report 2020. Koladiba: Officer of district health department plan and monitoring office, Amhara region, Ethiopia, 2020

17 Kefale T. Dembiya district health office annual report 2017. Koladiba: Officer of district health office, Amhara region, Ethiopia, 2017.

18 De Alwis WR, Pakirisamy P, Wai San L, et al. A study on hand contamination and hand washing practices among medical students. ISRN Public Health 2012;2012:1-5.

19 Hayes RJ, Bennett S. Simple sample size calculation for clusterrandomized trials. Int J Epidemiol 1999;28:319-26.

20 Bartram J, Ballance R. World Health Organization \& United Nations Environment Programme. In: Water quality monitoring : a practical guide to the design and implementation of freshwater quality studies and monitoring programs / edited by Jamie Bartram and Richard Ballance. London: E \& FN Spon, 1996. https://apps.who.int/iris/ handle/10665/41851

21 WHO. Guidelines for drinking-water quality. In: Surveillance and control of community supplies. 3. 2 edn. Geneva, 1997. www.who. int/water_sanitation_health/dwq/gdwqvol32ed.pdf

22 Unnebrink K, Windeler J. Sensitivity analysis by worst and best case assessment: is it really sensitive? Drug Inf J 1999;33:835-9.

23 World Health Organization(WHO). Laboratory quality management system: Handbook, 2011. Available: file:///C:/Users/HP/AppData/ Local/Temp/lams_en-2.pdf [Accessed 29 Oct 2020]. 